\title{
Inclusion of Quantum Confinement Effects in Self-Consistent Monte Carlo Device Simulations
}

\author{
R. W. KELSALL* and A. J. LIDSEY \\ Institute of Microwaves and Photonics, School of Electronic and Electrical Engineering, \\ University of Leeds, Leeds LS2 9JT, UK
}

\begin{abstract}
The design of Monte Carlo FET simulations is discussed, with specific attention to the methods used to describe quantum confinement effects. A new model is presented, which employs self-consistent coupling of Schrodinger, Poisson and Monte Carlo algorithms, and explicit calculation of the scattering rates between confined and unconfined states. Comparisons between the new model and a standard semi-classical Monte Carlo model are presented for a $0.1 \mu \mathrm{m}$ gate-length $\operatorname{In}_{0.52} \mathrm{Al}_{0.48} \mathrm{As} / \mathrm{In}_{0.53}$ $\mathrm{Ga}_{0.47} \mathrm{As} / \mathrm{InP}$ MODFET. Whilst the quantum model yields minor corrections in the predicted output characteristics, it is found that these results can be achieved without repeated iterations of the Schrodinger equation.
\end{abstract}

Keywords: Monte Carlo, HEMT, MODFET, quantum

\section{INTRODUCTION}

The methodology for developing semiconductor device simulations using Monte Carlo algorithms is now reasonably mature. The scheme of coupling an Ensemble Monte Carlo particle simulation to a 2-dimensional (2D) Poisson Solver has been successfully applied to both FETs and HBTs, and now represents the standard method in this subject area [1-2]. Numerous enhancements to the basic model have been developed to obtain greater accuracy-in particular the use of exact [3] or parameterised [4] 'full bandstructure' models.
However, the standard Monte Carlo device simulation is semi-classical: electrons and holes are modelled as discrete particles, whilst scattering between states is described using quantum mechanical perturbation theory. It has long been appreciated that in certain situations - such as the MOSFET inversion layer and the MODFET channel layer - quantum confinement effects may be significant. Inclusion of such effects requires, in principle, self-consistent solution of the 2D Poisson and Schrodinger equations, and the Monte Carlo transport algorithm; a task which appears far too computationally expensive for practical

\footnotetext{
* Corresponding author
} 
application. Consequently, there have been very few attempts to incorporate quantum confinement. In this paper, we review briefly some previous Monte Carlo FET models in which the problem has been addressed, and then discuss our own Monte Carlo/ Poisson/Schrodinger MODFET simulator.

\section{REVIEW OF MONTE CARLO FET MODELS}

Quantum confinement effects were actually considered in one of the earliest Monte Carlo MODFET models. Ravaioli and Ferry [5] calculated the first two quantised energy levels in the MODFET channel using a triangular well approximation. This yielded an analytic solution parameterised by the perpendicular (gate-substrate) electric fields thus, the energy level values could be easily updated when the Poisson equation was re-solved, and the effect of potential variation along the channel could be included. The scattering rates between quantum confined states were parameterised by energy level separation and carrier density, and hence could also be updated. Clearly, the main shortcoming of this otherwise elegant approach is the validity of the triangular well approximation these devices.

Park and Brennan [6] developed a MODFET simulator which also included solutions of the Schrodinger equation for the first two subbands in the channel. The eigenvalues were obtained from a numerical solution of the 1-dimensional (1D) Schrodinger equation, whilst the scattering rates were obtained using approximate variational wavefunctions. However, the Schrodinger equation was solved assuming an average $2 \mathrm{D}$ carrier density in the channel, hence no position dependence was included.

A key aspect of including quantum confinement in FET simulations is the method used for describing transitions between (quantum) confined and unconfined (classical or bulk-like) states. This problem is, itself, and artifact of the simulation model: in reality all states can be described using quantum mechanics; however, the use of Monte Carlo particle algorithm leads inevitably to a classical model of electron kinematics. In both the above models, electrons outide the channel were modelled as semi-classical particles. Electrons in the channel layer which acquired a kinetic energy in excess of the barrier height were also 'converted' into classical particles.

A similar scheme was used by Fischetti and Laux, in their simulation of inversion layer MOSFETs [7]. Their simulation code includes Monte Carlo, 2D Poisson and 1D Schrodinger algorithms, which are solved self-consistently. The scattering rates between confined states are calculated using the exact numerical wavefunctions. However, Fischetti and Laux also conclude, as above, that it is not practical to describe all electrons in the device by Schrodinger eigenfunctions. Hence their model, too, includes transitions between two alternative descriptions of electronic states. Scattering rates were calculated according to the nature of the initial state: for unconfined electrons, rates for transitions between pairs of bulk-like states were used, whereas, for electrons confined in the channel, rates for transitions between pairs of quantum confined states were used, irrespective of the energy of the final state. Such a scheme again necessitates 'conversion' of confined electrons into unconfined, and vice versa. These 'conversions' violate energy and momentum conservation, because the quantised spectrum of states in the channel has fewer degrees of freedom than the continuum to bulk states. Also, the unconfined 'conversion' process contained no physics to specify in which subband the 'converted' electron should reside.

\section{A NEW SELF-CONSISTENT SCHRODINGER-POISSON-MONTE CARLO MODEL}

Our MODFET model also includes self-consistently coupled Schrodinger, Monte Carlo and Poisson algorithms. However, in an attempt to overcome some of the problems associated with 
conversion between the confined and unconfined descriptions of electrons states, we have designed an alternative model of the scattering and kinematics involved in electron transfer in/out of the channel.

The Schrodinger equation is solved numerically in a series of $1 \mathrm{D}$ slices between source and drain, but self-consistency between the electrostatic potential and quantum-mechanical charge distribution is maintained. The scattering rates between confined states in the MODFET channel are calculated at each position along the channel using the exact numerical wavefunctions, and are recalculated periodically throughout the simulation.

Electrons outside the channel layer are modelled as classical particles. Within the channel layer, both particle-like electrons and quantum confined electrons are permitted. Quantum confined states are calculated for the first $n$ subbands in the channel: higher subbands are assumed to merge into a continuum, characterised by bulk-like states. Transitions between confined and continuum states are described solely by scattering processes. For confined-continuum scattering, the final state is described by a wavepacket which has a single k-value, a constant amplitude within the channel layer, and zero amplitude outside. A bulklike density of final states is assumed. After such a scattering event, the final state is modelled as a classical particle with a well defined energy and wavevector: its real space co-ordinate (in the direction prependicular to the heterolayers) is chosen randomly within the channel.

Conversely, for any particle which enters the channel there is a non-zero probability of scattering into a confined state. Transition rates are calculated using, for the initial state, a wavepacket as described above, and a Schrodinger eigenfunction for the final state. The final density of states is that appropriate to the quantum confined subband.

Transitions between confined and continuum states are mediated by all possible interactions: acoustic and polar optical phonon, and alloy disorder scattering. Whilst this approach avoids some of the difficulties with energy and momentum conservation discussed above, we have clearly deployed new approximations, such as the assumption of a contiunuous spectrum of states and the form of the continuum wavepacket. Our choice of wavepacket function appears to best reflect the bulk-like form of incoming and outgoing particles. No confinement effects are included for the $X$ and $L$ valleys, and scattering rates from confined $\Gamma$ states to the (unconfined) $X$ and $L$ valleys are calculated explicitly. In practice we have included just the lowest three subbands in the channel: a fourth subband can be included without too much computational expense, but its population is invariably small, and thus will have little effect on the simulation results.

\section{SIMULATION PERFORMANCE}

To test our simulation, we chose for simplicity a 3layer MODFET geometry. The materials system was lattice matched $\operatorname{In}_{0.52} \mathrm{Al}_{0.48} \mathrm{As} / \mathrm{In}_{0.53} \mathrm{Ga}_{0.47} \mathrm{As} /$ InP, which has large conduction band offsets and is therefore more likely to be affected by quantum confinement. No cap layer was included in the simulation: injection and collection were modelled using ohmic source and drain regions which spanned both the supply and channel layers (see Fig. 1).

Our first objective was to ascertain the importance of quantum confinement by comparing results of our new code with those from our semi-classical Monte Carlo model. On a microscopic level, we may expect two principal differences: in the distribution of charge across the channel width, and in the electron scattering rates. Figure 2 shows a comparison of the scattering

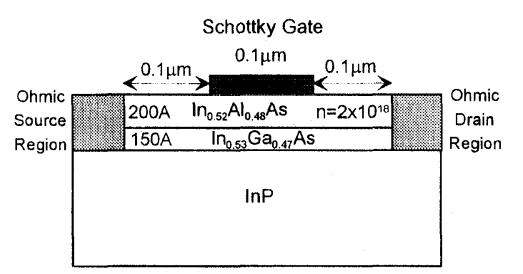

FIGURE 1 Schematic diagram of the simulated $0.1 \mu \mathrm{m}$ gatelength InP MODFET. 


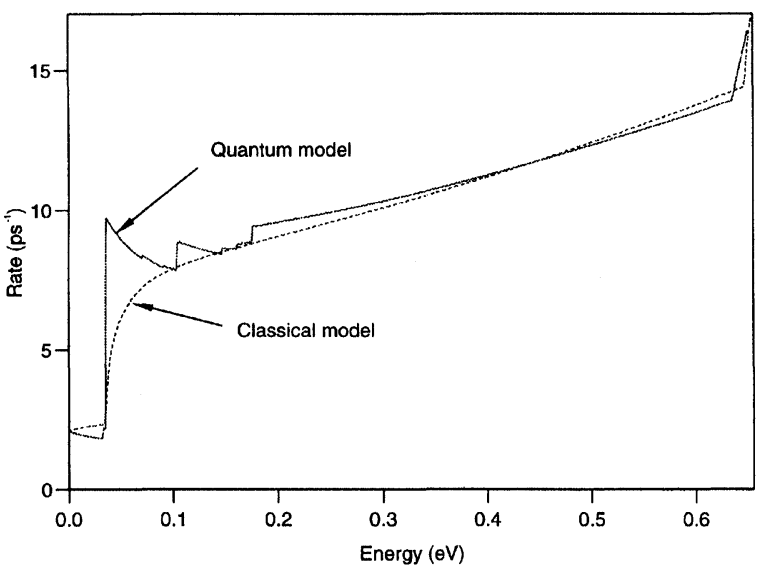

FIGURE 2 Full curve: total (phonon plus alloy) scattering rate for an electron in the lowest subband in the channel. (The impurity scattering rate was not included in this diagram since, although the rates (at low electron energies) are large, the effect of impurity scattering is relatively small, as it gives no energy relaxation and relatively little momentum relaxation). The electron wavefunctions were calculated at the mid-point of the channel, for gate and drain biases of $V_{\mathrm{G}}=0.2 \mathrm{~V}, V_{\mathrm{D}}=1.0 \mathrm{~V}$. Broken curve: scattering rate calculated in the standard semiclassical model.

rates for the two models. The rates are very similar at high energies (the shoulder at $E \approx 0.65 \mathrm{eV}$ marks the onset of intervalley scattering, which has identical rates in both models). At low energies the total rate in the quantum model exceeds that in the classical model, due to the difference in the $2 \mathrm{D}$ and $3 \mathrm{D}$ densities of states.

Figure 3 shows the charge distrubution at the source end of the channel in the quantum and classical simulations. The delocalised nature of the electron wavefunction results in a broader charge distribution in the quantum model, with much less charge accumulation at the channel/supply layer interface $(d=200 \AA)$ - where the channel potential is lowest.

In Figures 4-6 we examine the extent of channel non-uniformity. Figure 4 shows typical potential profiles taken from the source, gate and drain regions of the device. Figure 5 shows the wavefunctions for the first eigenstate at the same three locations. There is very little difference in the wavefunctions in the source and drain regions, despite the much lower potential at the drain end

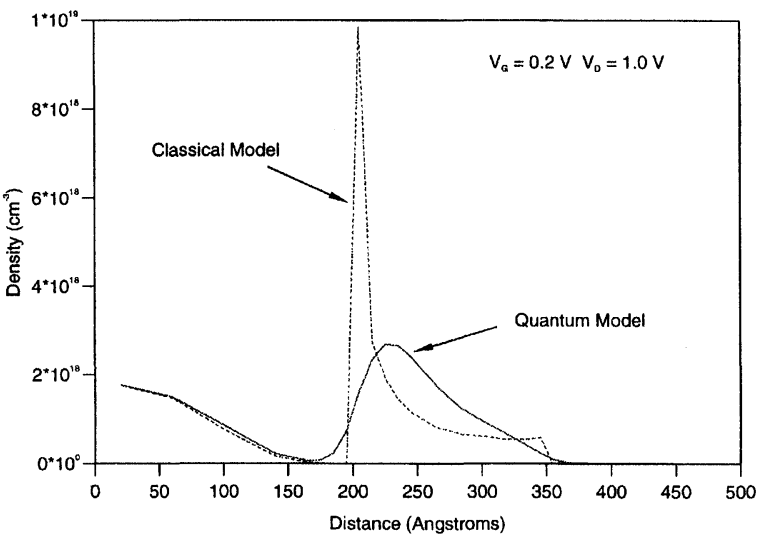

FIGURE 3 Electron distribution across a vertical slice in the source region of the channel. The horizontal scale measures distance from the top of the InAlAs supply layer downwards towards the substrate, with the channel located between $d=200-350 \AA$.

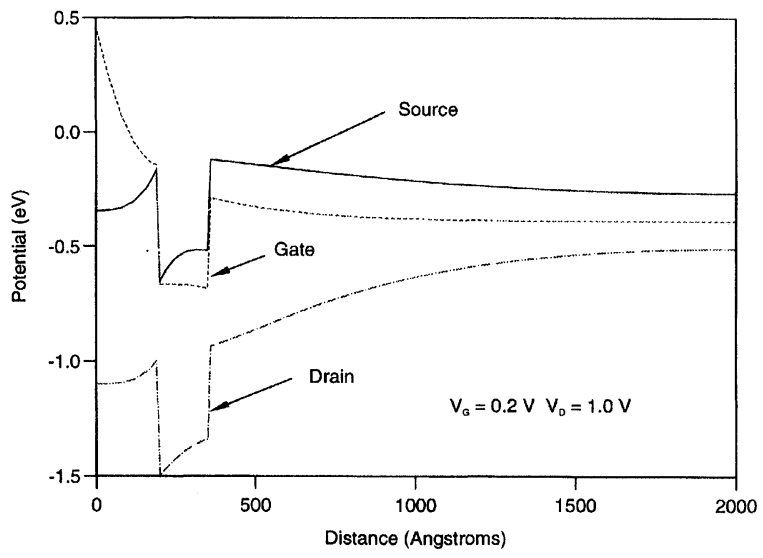

FIGURE 4 Potential (conduction band-edge) profiles along vertical slices taken in the source, gate and drain regions respectively. Horizontal scale as in Figure 2.

of the device, but the peak in the wavefunction under the gate is shifted, due to the altered shape of the channel potential well. However, Figure 6 shows that the shift in wavefunction position leads to only a few percent difference between the confined-confined scattering rates at different positions along the channel. For this bias configuration the same observations apply to scattering from higher subbands too: thus it appears that channel non-uniformity has relatively little effects on scattering. 


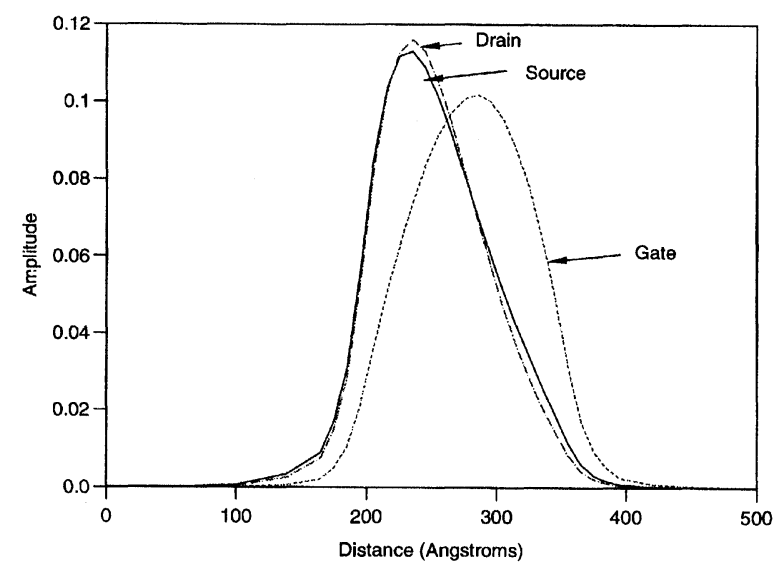

FIGURE 5 Wavefunctions of the lowest eigenstate in the source, gate and drain regions of the device: $V_{\mathrm{G}}=0.2 \mathrm{~V}$ $V_{\mathrm{D}}=1.0 \mathrm{~V}$.

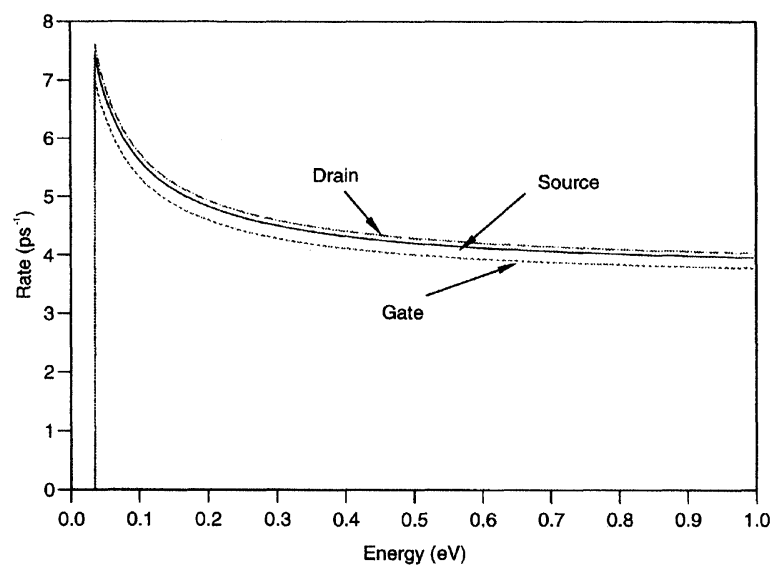

FIGURE 6 Confined-confined (intrasubband) polar optical phonon scattering rate for an electron in the lowest subband, in the source, gate and drain regions of the channel. $V_{\mathrm{G}}=0.2 \mathrm{~V}$, $V_{\mathrm{D}}=1.0 \mathrm{~V}$.

The main computational overhead in a selfconsistent simulation is the frequency at which the relevant parameters must be re-calculated. In Figures 7 and 8 we compare wavefunctions and scattering rats from simulations with minimum and maximum self-consistency. In both cases the simulation was run using the classical model, until a steady state was attained. In the minimum update case, the Schrodinger equation was then solved once, at each position along the channel, using a time-averaged potential. The channel

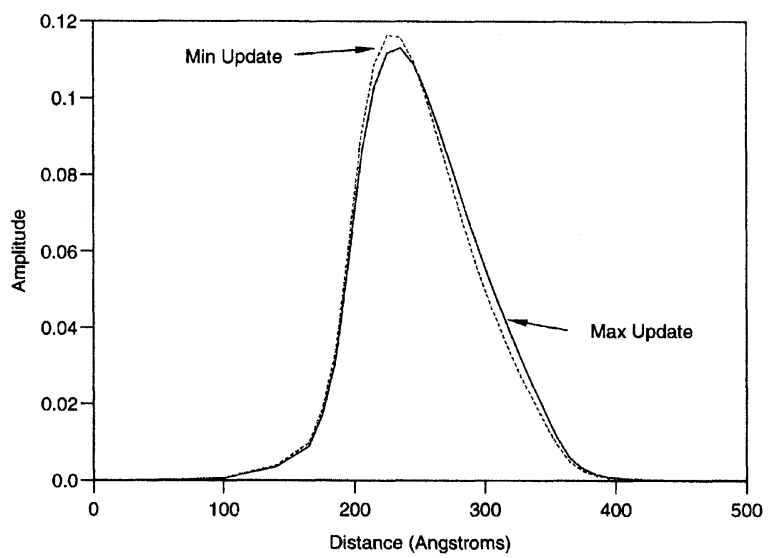

FIGURE 7 Ground-state wavefunction at the source end of the channel, obtained using minimum and maximum updates (see text). $V_{\mathrm{G}}=0.2 \mathrm{~V}, V_{\mathrm{D}}=1.0 \mathrm{~V}$.

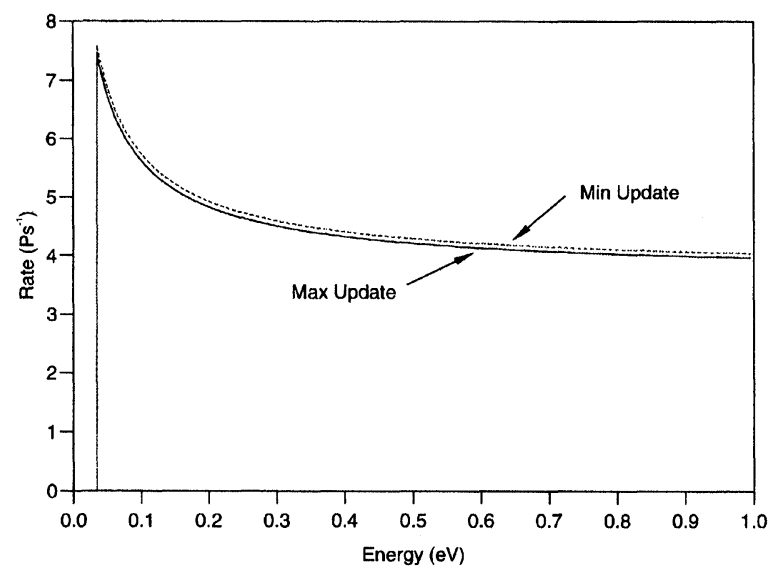

FIGURE 8 Confined-confined (intrasubband) polar optical phonon scattering rate for an electron in the lowest subband, at the source end of the channel, obtained using minimum and maximum updates. $V_{\mathrm{G}}=0.2 \mathrm{~V}, V_{\mathrm{D}}=1.0 \mathrm{~V}$.

scattering rates were calculated using the Schrodinger wavefunctions. The simulation was then continued, using the quantum model, but with no re-calculation of the channel eigenstates or scattering rates. In the maximum update case, the simulation was continued, re-solving the Schrodinger equation at every Poisson timestep. The channel scattering rates were calculated using the original set of eigenstates, and then on a further five occasions during the simulation, using timeaveraged eigenstates in each case. 


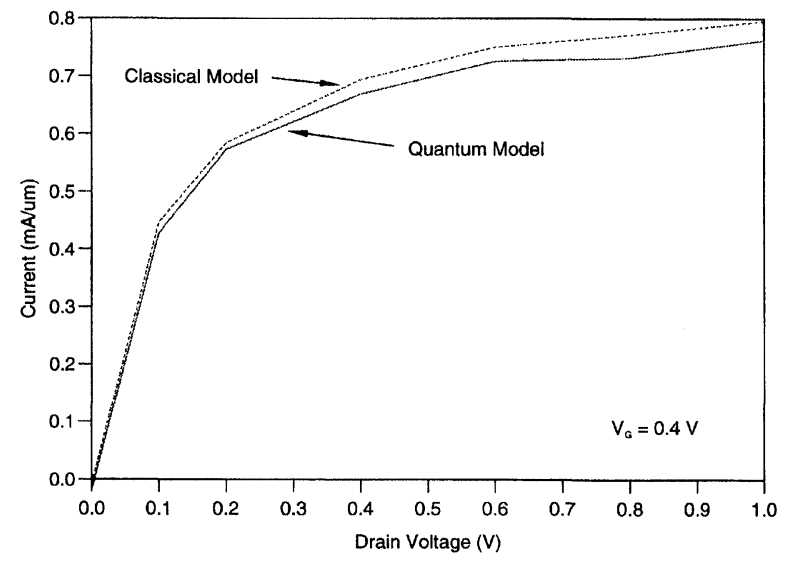

FIGURE 9 Simulated output characteristics for the $0.1 \mu \mathrm{m}$ gate-length InP MODFET using the classical and quantum models.

In practice, we found surprisingly little difference between the steady-state wavefunctions for the two cases. Figure 7 is typical of all the comparisons we examined, for wavefunctions at different positions and for different subbands. Correspondingly, the scattering rates show little variation with update frequency; again, Figure 8 is typical of the various rates we examined for this device geometry and biasing.

Figure 9 shows that the quantum model predicts a reduced drain current throughout the bias range: this is caused by a reduction in the predicted electron velocities, which is partly due to the greater phonon scattering (as discussed above). However, it must also be appreciated that the quantum model includes remote impurity scattering, a concept which does not feature in the classical description, and which provides some additional momentum relaxation at low energies. Despite the difference in charge distribution in the quantum and classical models, the overall electron density in the channel is very similar.

\section{CONCLUSIONS}

Our aim in this work has been to examine the importance of including quantum confinement effects in Monte Carlo MODFET simulations.
Clearly, we have yet to cover the full parameter space for such devices: however, our results to date imply that the inclusion of confined electron states, and of quantum confined scattering rates, has only a modest effect on predictions of device performance, relative to those obtained from standard semi-classical Monte Carlo models. Whilst the quantum model predicts, in general, a broader charge density distribution across the channel than does the calssical model, the overall charge density in the channel is largely unchanged. Furthermore, it appears that the corrections predicted by the quantum model can be obtained without recourse to a fully self-consistent Schrodinger/Poisson/ Monte Carlo scheme: once a steady state solution is obtained from the classical model, the quantum correction can be obtained by a single calculation of the channel eigenstates and corresponding wavefunctions. This represents a substantial saving in CPU time.

\section{References}

[1] Hockney, W. and Eastwood, J. W. (1988). Computer Simulation Using Particles IOP Publishing Ltd., Bristol.

[2] Jacoboni, C. and Lugli, P. (1989). The Monte Carlo Method for Semiconductor Device Simulation Springer-Verlag, Vienna.

[3] Fischetti, M. V. and Laux, S. E. (1988). "Monte Carlo analysis of electron transport in small semiconductor devices including band-structure and space-charge effects", Phys. Rev. B., 3, $9721-9745$.

[4] Venturi, F., Sangiorgi, E., Brunetti, R., Quade, W., Jacoboni, C. and Ricco, B. (1991). "Monte Carlo simulations of high-energy electrons and holes in Si-n-MOSFETs", IEEE Trans. Computer Aided Design, 11, 1276-1286.

[5] Ravaioli, U. and Ferry, D. K. (1986). "MODFET Ensemble Monte Carlo model including the quasi-twodimensional electron gas", IEEE Trans. Electron Devices, 33, 677-681.

[6] Park, D. H. and Brennan, K. F. (1990). "Monte Carlo simulations of 0.35 um gate length $\mathrm{GaAs}$ and InGaAs HEMTs", IEEE Trans. Electron Devices, 37, 618-628.

[7] Fischetti, M. V. and Laux, S. E. (1993). "Monte Carlo study of electron transport in silicon inversion layers", Phys. Rev. B., 48, 2244.

\section{Authors' Biography}

Robert W. Kelsall was born in Rotherham, England in 1964. He received the B.Sc. and Ph.D. degrees from the University of Durham, 
UK, in 1985 and 1989 respectively; the latter for research in Monte Carlo modelling of electronic transport in GaAs quantum wells.

From 1989 to 1993 he worked as a research assistant, developing self-consistent Monte Carlo simulations of HEMTs and multilayer MOSFETs. $\mathrm{He}$ was appointed to a lectureship at the Uni- versity of Leeds, UK in 1993, and is a member of the newly formed Institute of Microwaves and Photonics at Leeds, where he is working on semiconductor device simulations for hot-carrier degradation studies in MOSFETs, quantum confinement effects in HEMTs, and far-infra-red intersubband lasers. 

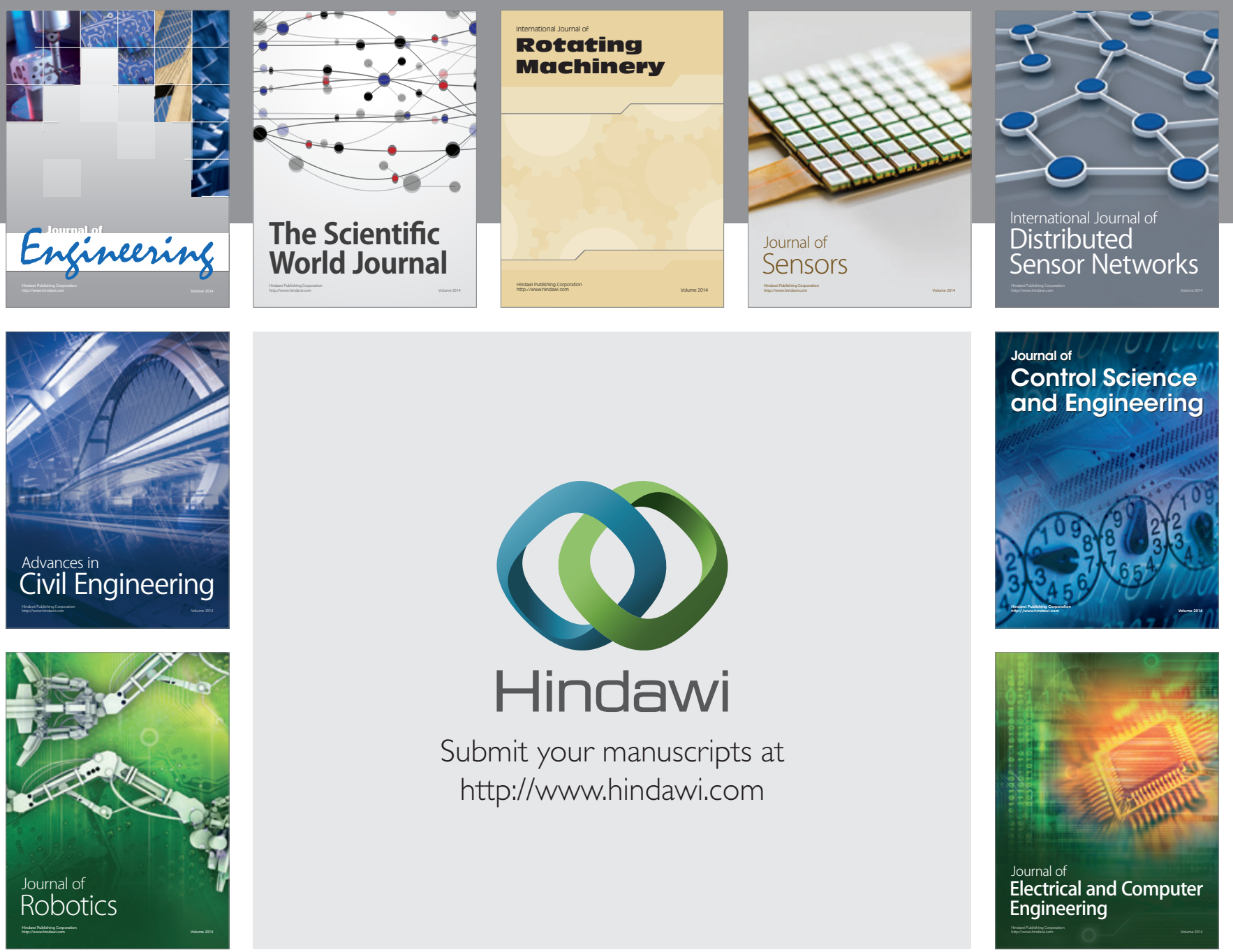

Submit your manuscripts at

http://www.hindawi.com
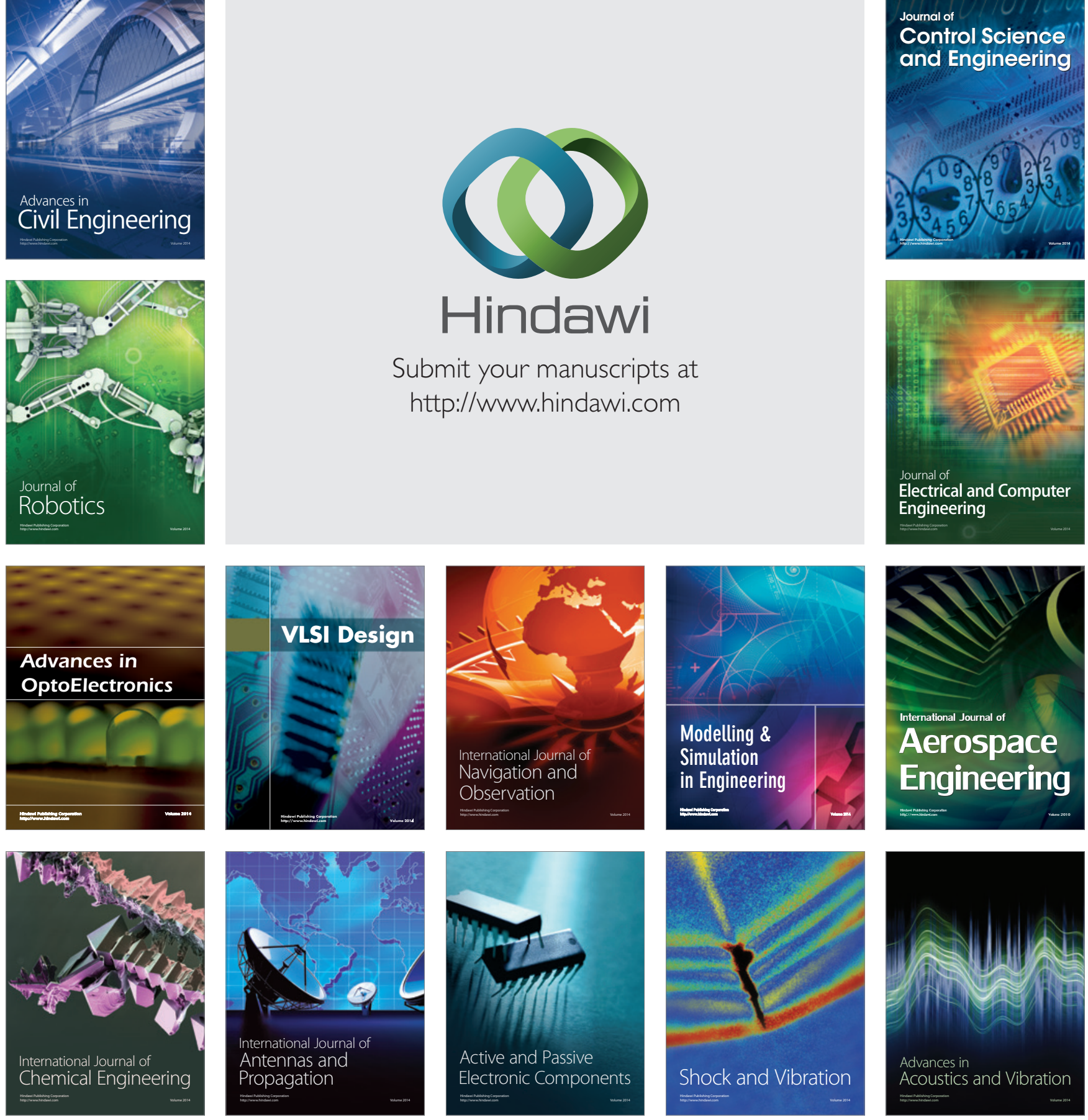